\title{
ALGUNAS NOTAS SOBRE EL LÉXICO MÉDICO EN LA NUEVA ESPAÑA DIECIOCHESCA: VOCES CULTAS Y POPULARES EN LA DENOMINACIÓN DE LAS ENFERMEDADES ${ }^{1}$
}

\author{
Some Notes About Medical Vocabulary in $18^{\text {th }}$ \\ Century New Spain: Technical and Colloquial Words \\ for the Denomination of Illnesses
}

José Luis RAMÍREZ LUENGO

Universidad Autónoma de Querétaro

jose_ramirez14@hotmail.com

Fecha de recepción: 19/11/2014

Fecha de aceptación definitiva: 13/03/2015

RESUMEN: Si bien el léxico de la medicina dieciochesca es algo que se ha venido estudiando durante los últimos años en España, no ocurre lo mismo en América, donde las aproximaciones a este tema son todavía notablemente escasas. En este caso, se pretende estudiar las denominaciones que, para referirse a distintas enfermedades, se descubren en una colección documental dieciochesca de Nueva España: en concreto, se procederá a describir el corpus para después analizar los vocablos que aparecen en los textos, teniendo en cuenta cuestiones como los neologismos, el uso de vocablos fluctuantes y la presencia de voces populares. Los propósitos del trabajo, por tanto, son tres: 1) evidenciar la importancia de la

1. Este trabajo se encuadra en el proyecto Estudio del vocabulario científico y técnico en el español del siglo XVIII. Ciencia y técnica en América (VCT18II), dirigido por la Profa. Gómez de Enterría (Universidad de Alcalá), y financiado por el Ministerio de Economía y Competitividad (ref.: FFI2011-24090). 
documentación notarial para el estudio del léxico médico; 2) aportar datos que contribuyan a escribir la historia de este léxico en la Nueva España; y 3) señalar vías de análisis que puedan explorarse en posteriores trabajos.

Palabras clave: historia de la lengua; léxico; medicina; Nueva España; siglo XVIII; voces populares.

ABSTRACT: Whereas the $18^{\text {th }}$ Century medical vocabulary is something that has been studied during recent years in Spain, the situation is very different in Latin America, where papers on this subject are very limited. In this case, this paper aims to study the denominations for illnesses that were discovered in a $18^{\text {th }}$ Century New Spain document corpus: to do so, the corpus will be described and then the vocabulary used in the documents will be analysed; the paper will pay special attention to questions such as neologisms, fluctuating words and the presence of colloquial vocabulary. Thus, the purposes of the paper are three: 1) to demonstrate the importance of official documents for the study of medical vocabulary; 2) to provide some data for writing the history of this vocabulary; and 3) to note some analyses that should be done in the future.

Key words: History of the Spanish language; Vocabulary; Medicine; New Spain; $18^{\text {th }}$ Century; Colloquial words.

1. No cabe duda de que el estudio del vocabulario empleado en la práctica médica del siglo XVIII constituye aún un amplio campo de trabajo para los investigadores interesados en la historia del léxico español: así, si bien es de justicia señalar la existencia de estudios que sirven de marco explicativo general ${ }^{2}$ y otros más específicos en los que se lleva a cabo el análisis de vocablos concretos o su aparición en determinadas obras lexicográficas ${ }^{3}$, lo cierto es que todavía son

2. Álvarez de Miranda, Pedro. Palabras e ideas. El léxico de la Ilustración temprana en España. Madrid: Real Academia Española, 1992; Álvarez De Miranda, Pedro. "El léxico español, desde el siglo XVIII hasta hoy». En CANO, Rafael (coord.). Historia de la lengua española. Barcelona: Ariel, 2004, pp. 1037-1064.

3. GómEZ DE ENTERRÍA, Josefa. "El vocabulario médico de los novatores en el siglo XVIII». En García Godoy, María Teresa (ed.). El español del siglo XVIII. Cambios diacrónicos en el primer español moderno. Berna: Peter Lang, 2012, pp. 55-81; GómEZ DE ENTERRÍA, Josefa. "El vocabulario de la medicina del siglo XVIII a través de las traducciones de los hermanos Juan y Félix Galisteo Xiorro». En MONTERO CARTElle (ed.). Actas del VIII Congreso Internacional de Historia de la Lengua Española, II. Santiago de Compostela: Meubook, 2012, pp. 1405-1422; GómEZ DE ENTERRía, Josefa y Natividad GALLARDO. "Las versiones de medicina y botánica y la nueva terminología científica en el siglo XVIII". Cuadernos del Instituto de Historia de la Lengua, 2010, 4, pp. 55-75; GutiérREz RODILLA, Bertha M. "Los términos relacionados con la medicina en el Diccionario de Autoridades". Boletín de la Real Academia Española, 1993, 73/260, pp. 463-512; GuTIÉRREz RODILlA, Bertha M. «Construcción y fuentes utilizadas para los términos médicos en el Diccionario de Autoridades». Revista de Lexicografia, 1, 1994-5, pp. 149-162; Gutiérrez Rodilla, Bertha M. "El léxico de la medicina en el diccionario de Esteban Terreros y Pando". 
muchas y muy variadas las cuestiones que los estudiosos deben analizar al respecto para que se pueda obtener una visión más o menos completa del léxico que utilizan los profesionales de la medicina en la Centuria Ilustrada.

Quizá una de esas cuestiones hasta el momento desatendidas -y que, afortunadamente, están dejando de serlo- sea todo aquello que tiene que ver con la diatopía de tales usos, y más específicamente con el vocabulario empleado en la medicina de las amplias regiones que constituyen Hispanoamérica: en efecto, una rápida revisión de los títulos anteriormente citados demuestra la concentración de los análisis en el ámbito peninsular, y el total abandono de las variedades americanas del español ${ }^{4}$. Tal situación, sin embargo, está empezando a cambiar desde hace poco tiempo, y a día de hoy se cuenta ya con estudios muy recientes que analizan diversos aspectos del léxico empleado en la medicina americana dieciochesca, algunos sobre asuntos tan principales en la época como las fiebres 5 , la higiene ${ }^{6}$ o la viruela ${ }^{7}$, por más que es aún mucho lo que queda por analizar acerca de esta cuestión.

Resulta, pues, fundamental continuar con el inventariado y el análisis del vocabulario médico de la América del siglo XVIII por medio del expurgo de la documentación de todo tipo generada en esta región y de la incorporación de los datos extraídos de tales búsquedas en un marco general que explique sus características y especificidades, pues solo así se podrá obtener una visión más completa

En Alonso González, Alegría et al. (eds.). Actas del III Congreso Internacional de Historia de la Lengua Española, II. Madrid: ArcoLibros, 1996, pp. 1327-1342.

4. No puede por menos que sorprender tal situación si se tiene en cuenta no solo el interés intrínseco del análisis en sí, sino también la importancia que tal análisis puede tener para la mejor y más completa comprensión de los hechos registrados en España, habida cuenta de que «el estudio del léxico que se utiliza en la práctica médica durante el siglo XVIII americano permite no solo establecer las divergencias y semejanzas que, a este respecto, existen en ambas zonas geográficas, sino también analizar - a partir de la constatación de este hecho- cuestiones varias como las especificidades propias que presenta cada una de las zonas o las relaciones que tales zonas pueden establecer entre sí, entre otras muchas» (RAmírez Luengo, José Luis. "Un aporte a la historia del léxico médico en América: el vocabulario de la viruela en la Nueva España dieciochesca». Anuario de Letras. Lingüística y Filología, 2014; II/1, en prensa). No sorprende, por tanto, que el estudio de esta cuestión -en el marco más amplio del estudio del léxico científico dieciochesco en el Nuevo Mundo- se haya definido como "una de las labores más urgentes a las que se enfrenta actualmente la investigación diacrónica del español» (RAmírez LuENGO, José Luis. "Aproximación al léxico de la medicina en el Buenos Aires del siglo XVIII". En Actas del IX Congreso Internacional de Historia de la Lengua Española; en prensa).

5. Gómez de EnTERría, Josefa. "El vocabulario de las fiebres epidémicas en español del siglo XVIII». En Ramírez Luengo, José Luis y Velásquez Upegui, Eva Patricia (eds.). La historia del español boy. Estudios y perspectivas. Lugo: Axac (en prensa).

6. Gómez DE ENTERRÍA, Josefa. "Higiene y salud en las traducciones médicas del francés al español durante el siglo XVIII». Panace@. Revista de medicina, lenguaje y traducción, 14/38, 2013, pp. 287-295.

7. Ramírez Luengo, José Luis. «Un aporte a la historia del léxico médico en América: el vocabulario de la viruela en la Nueva España dieciochesca». Anuario de Letras. Lingüústica y Filología, II/1, 2014 (en prensa). 
y más realista del proceso histórico que determina la configuración de un léxico especializado para la práctica de la medicina no tanto en variedades concretas, sino en el ámbito general de la lengua española.

2. Teniendo en cuenta lo anterior, parece necesario preguntarse ahora qué corpus se puede emplear a la hora de estudiar el léxico médico empleado en América -O, más en concreto, en la Nueva España- a lo largo del siglo XVIII; por supuesto, siendo la medicina y la salud una materia que permea prácticamente todos los campos y momentos de la vida, es muy probable que textos tipológicamente muy variados puedan resultar de interés para recolectar las voces relacionadas con la práctica médica, y tal aserto queda confirmado en la descripción del corpus de estudio que emplean en su proyecto de investigación sobre este asunto Gómez de Enterría y Gallardo ${ }^{8}$, en el que se presenta una extensísima nómina de materiales que van desde los tratados científicos de autores españoles hasta «epistolarios y obras literarias que abordan de alguna manera temas relacionados con la enfermedad y la salud", pasando por traducciones, textos docentes o la misma prensa periódica.

Considerando, por tanto, lo inmediatamente indicado, en este caso concreto -y a consecuencia de los buenos resultados obtenidos en el estudio del léxico de la viruela ${ }^{9}-$, se ha optado por utilizar como corpus de estudio un conjunto de documentos notariales ${ }^{10}$ generados en diversas partes del Virreinato de la Nueva España (y, más en concreto, en diversos territorios de lo que hoy constituye México) a lo largo de la segunda mitad del siglo XVIII, muy especialmente en los últimos años de la centuria; así, se trata de un total de 60 documentos de diversa longitud que, en su práctica totalidad, se guardan en los distintos volúmenes que componen la sección Epidemias (044) del Archivo General de la Nación (México D. F., México $)^{11}$, en la que -como queda claro por su nombre- se conserva la documentación que produce la administración colonial a la hora de enfrentarse a las distintas epidemias que afectan al territorio durante los siglos XVI, XVII y XVIII ${ }^{12}$.

8. GómEZ DE ENTERRía, Josefa y Natividad GALLARDO. «Las versiones de medicina y botánica y la nueva terminología científica en el siglo XVIII". Cuadernos del Instituto de Historia de la Lengua, 2010, 4, pp. 55-75; pp. 57-63.

9. RAmírez Luengo, José Luis. "Un aporte a la historia del léxico médico en América: el vocabulario de la viruela en la Nueva España dieciochesca». Anuario de Letras. Lingüística y Filología, 2014, II/1, (en prensa).

10. Una vez más, se hace necesario indicar aquí que se emplea el término notarial de una manera notablemente laxa, con el significado de 'texto de carácter administrativo escrito -generalmente, aunque no de manera obligatoria- por un escribano o notario'.

11. En concreto, los volúmenes 1, 3, 5, 6, 7, 10, 11, 15, 16 y 17; así mismo se han considerado cuatro documentos que no se conservan en esta sección, sino en Indiferente Virreinal/Caja 1807, donde también se guarda documentación relacionada con las epidemias que afectan al territorio novohispano durante el siglo XVIII.

12. El interés de una documentación como la presente para el tema que se pretende trabajar resulta, pues, manifiesto por muy diversos motivos: más allá de que se trate de documentación especí- 
Por otro lado, la disparidad de asuntos a los que se enfrenta la administración colonial y la necesidad de atenderlos de la forma más eficaz posible determina la aparición de una multiplicidad de tipos textuales diferentes y, con ello, de una enorme diversidad tipológica que se engloba -y en cierto modo queda ocultadentro del término notarial empleado más arriba; es necesario precisar, por tanto, que el corpus empleado en el estudio no es homogéneo desde este punto de vista, sino que cuenta en su seno con documentos tan variados como pueden ser, por ejemplo, memoriales de procedencia pública y privada, informes administrativos, reconocimientos médicos o dictámenes que diversos profesionales de la medicina hacen a instancias de las instituciones virreinales acerca de tratados científicos o cuestiones asistenciales ${ }^{13}$, es decir, con «textos de muy distinto carácter y, así mismo, de diferente funcionalidad, lo que conlleva la aparición de muchas y muy diversas voces relacionadas con la práctica médica ${ }^{14} \mathrm{y}$, al mismo tiempo, pone de manifiesto la importancia de tales materiales de archivo para un estudio como el presente.

3. Una mera aproximación superficial a los documentos que componen el corpus permite comprobar con facilidad dos cuestiones de sumo interés para un análisis como el que se pretende llevar a cabo en estas páginas: por un lado -y según se dijo anteriormente-, la profusión y la abundancia de términos relacionados con la medicina que se puede registrar en ellos, y que alcanza sin ninguna dificultad más del centenar ${ }^{15}$; por otro, la enorme cantidad de autores que generan estos documentos y su disparidad en cuanto a la formación que poseen, pues si

ficamente dedicada a temas médicos y, por ello, riquísima en vocabulario propio de la disciplina, cabe señalar otros factores como, por ejemplo, su relativa abundancia, así como, desde un punto de vista metodológico, tres características fundamentales para la Dialectología Histórica ya señaladas en Ramírez Luengo (RAmírez Luengo, José Luis. "Aproximación al léxico de la medicina en el Buenos Aires del siglo XVIII". En Actas del IX Congreso Internacional de Historia de la Lengua Española; en prensa): 1) ser generada por personas originarias de la zona o que, al menos, desarrollan su vida en este lugar; 2) reflejar el vocabulario -en este caso, médico- que efectivamente se emplea en la zona en cuestión; y 3) estar rigurosamente fechada.

13. Se trata, por tanto, de documentos que, de acuerdo con la clasificación tipológica llevada a cabo en Ramírez Luengo (RAmírez Luengo, José Luis. "Aproximación al léxico de la medicina en el Buenos Aires del siglo XVIII". En Actas del IX Congreso Internacional de Historia de la Lengua Española; en prensa), se encuadran dentro de los puntos 1.2. (documentos derivados de la práctica médica -reconocimientos médicos), 2.1.1.1. (documentos administrativos de contenido médico de carácter público -informes/memoriales de procedencia pública) y 2.1.2.1. (documentos administrativos de contenido médico de carácter privado-memoriales de procedencia privada).

14. RAmírez Luengo, José Luis. «Un aporte a la historia del léxico médico en América: el vocabulario de la viruela en la Nueva España dieciochesca». Anuario de Letras. Lingüística y Filología, 2014, II/1, (en prensa).

15. Piénsese que solo en el caso de la viruela -y más en concreto, de los tipos de la enfermedad, de sus fases y de las denominaciones dadas a los enfermos- se registra un total de 36 unidades (RAMíREZ LuEngo, José Luis. "Un aporte a la historia del léxico médico en América: el vocabulario de la viruela en la Nueva España dieciochesca». Anuario de Letras. Lingüística y Filología, 2014, II/1 (en prensa). 
muchas veces son facultativos y profesionales de la medicina (a manera de ejemplo, los diagnósticos recogidos en AGN Epidemias (044), vol. 3/Exp. 1/f. 42; vol. 5/Exp. 2/f. 62; vol. 7/Exp. 1/f. 87), en no pocos casos se trata de religiosos y/o de personal relacionado con la administración colonial (entre otros, AGN Epidemias (044), vol. 3/Exp. 2/f. 81), esto es, de individuos que en ocasiones se pueden acercar a la práctica médica, pero en general se pueden englobar más bien en el grupo de los no especialistas en esta cuestión.

Esta última circunstancia resulta, precisamente, de primera importancia si, junto a las voces más técnicas, se pretende acceder a vocablos de carácter si se quiere más popular, es decir, que están relacionadas con lo que Gómez de Enterría y Gallardo ${ }^{16}$ denominan «el léxico de los pacientes», entendido como el vocabulario con el que el profano hace referencia a la experiencia de la enfermedad y/o la medicina en su vida cotidiana, y que muy difícilmente tiene cabida en los tratados científicos, los textos docentes o todas aquellas obras que reflejan el discurso médico académico y especializado; de este modo, el empleo de la documentación notarial como corpus en los estudios históricos acerca del léxico de esta disciplina se caracteriza sin ninguna duda por las posibilidades que abre para la detección y el análisis tanto del vocabulario consagrado por los especialistas como de aquel que emplean los grupos de no-especialistas -aunque en ocasiones se puedan dedicar a la práctica médica-, que, por motivos más que evidentes, no son en modo alguno ajenos a la realidad de la enfermedad y de su sanación.

3.1. Por lo que se refiere al léxico que -de forma más o menos imprecisase puede denominar culto, es necesario señalar que su empleo resulta sin duda predominante en el corpus, consecuencia, evidentemente, de la presencia de médicos y otros facultativos entre los autores de los documentos y de la necesidad de estos de referirse a las dolencias sin ningún tipo de ambigüedad y, por tanto, por medio de tecnicismos altamente especializados. Tal estado de cosas se refleja, por ejemplo, en el empleo de voces como tos ferina, alferecía o los préstamos petequia, del italiano, y pleuresía, de origen galo (ejemplos 1-4), pero también en la presencia de compuestos cultos que matizan de forma precisa la enfermedad y sus síntomas -como muestra, fiebre gástrico-nerviosa o pútridomaligna (ejemplo 5)-, o en la utilización de derivados que se forman a partir de unos sufijos claramente privilegiados como son el antiguo participio de presente - entis ${ }^{17}$-con adjetivos como putrescente, febricitante, emoliente, convalenciente o el sustantivo intumescencia (ejemplos 6-10)- y muy especialmente -oso, que

16. GÓMEZ DE ENTERRÍA, Josefa y Natividad GALlARDO. "Las versiones de medicina y botánica y la nueva terminología científica en el siglo XVIII. Cuadernos del Instituto de Historia de la Lengua, 2010, 4, pp. 55-75; p. 58.

17. GÓmEZ DE ENTERRía, Josefa. «El vocabulario de las fiebres epidémicas en español del siglo XVIII». En Ramírez Luengo, José Luis y Velásquez Upegui, Eva Patricia (eds.). La historia del español boy. Estudios y perspectivas. Lugo: Axac (en prensa). 
Gómez de Enterría $^{18}$ considera un mecanismo muy fecundo para la formación de adjetivos en el léxico de la medicina de la Centuria Ilustrada y que en este corpus novohispano se descubre, por ejemplo, en los términos aceitoso, bilioso, cancroso, contagioso, gangrenoso, morboso, nauseoso, oleoso, poroso, varioloso/ viroloso, viscoso y vejigoso (ejemplos 11-13) ${ }^{19}$.

1. Pocos se morían en la curación, los más en la convalecencia, por quedarles una tos ferina, que presto los desaparecía (Chalco, 01/04/1797; vol. 3/Exp. 2/f. 88v. $)^{20}$.

2. En el primer tiempo que es el de el contagio, luego que he observado los síntomas unívocos o patognomónicos que son: la cardialgia o dolor de estómago (...) y convulsiones o alferecías en los niños (Campeche, 19/03/1793; vol. 7/Exp. 8/f. 332r.).

3. Le están brotando unas variolas malignas, y entre estas unas petequias negras, la que pienso se morirá (Orizaba, ?/05/1797; vol. 5/Exp. 2/f. 169r.).

4. Observé que la enfermedad epidémica ha sido una verdadera pleuresía seca (vulgarmente dolor de costado) (Zimapán, 06/02/1794; vol. 17/Exp. 1/f. 58r.).

5. Sigue la calentura que unos llaman gástrico-nerviosa, otros gástricopútrida otros pútrido-maligna etc., como se declaró en la junta que Su Señoría se sirvió mandar celebrar en la tarde del jueves (Veracruz, 01/03/1809; vol. 17/Exp. 7/f. 184r.).

18. GómEz DE ENTERría, Josefa. "El vocabulario médico de los novatores en el siglo XVIII». En García Godoy, María Teresa (ed.). El español del siglo XVIII. Cambios diacrónicos en el primer español moderno. Berna: Peter Lang, 2012, pp. 55-81; pp. 68-71.

19. La localización de los términos no ejemplificados a continuación es la siguiente: aceitoso, vol. 15/Exp. 4/f. 96r.; bilioso, vol. 11/Exp. 2/f. 151r.; contagioso, vol. 7/Exp. 1/f. 87r.; gangrenoso, vol. 11/Exp. 2/f. 138r.; morboso, vol. 5/Exp. 2/f. 67r.; oleoso, vol. 15/Exp. 4/f. 96r.; poroso, vol. 7/Exp. 8/f. 333v.; viscoso, vol. 7/Exp. 8/f. 333v.; vejigoso, vol. 7/Exp. 1/f. 87r. Cabe señalar, por otro lado, que ni viroloso ni cancroso aparecen recogidos hoy en el DRAE (REAL ACADEMIA EsPAÑola. Diccionario de la Lengua Española. $22^{\mathrm{a}}$ edición. Madrid: Espasa-Calpe, 2001), por más que este último aparece utilizado ya en el siglo XVII y, con cierta frecuencia, en el Siglo de las Luces (REAL Academia EsPañola. Fichero General de la Real Academia Española. En red: <http://www.rae.es>, 2014; s.v. cancroso); viroloso, por su parte, aparece en el repositorio académico como voz propia de regiones peninsulares tan dispares como Salamanca, Aragón, León o La Mancha (REAL ACADEMIA EsPañola. Fichero General de la Real Academia Española. En red: <http://www.rae.es>, 2014; s.v. viroloso).

20. Habida cuenta de que el interés de los ejemplos es meramente lexicológico, se ha optado por modernizar la grafía de ellos según las normas ortográficas actuales. Cabe indicar, por otro lado, que -a menos de que se indique lo contrario- todos están tomados de la sección Epidemias del Archivo General de la Nación (México) (AGN, Epidemias (044)), de manera que, tras el ejemplo en cuestión, se indica únicamente el volumen, el expediente y el folio donde aparece la voz que interesa en cada ocasión. 
6. De la implantación de partículas cáusticas, cancrosas y acérrimas en cuerpos afectos de humores putrescentes (...) resultó una combinación pésima (México, 25/02/1793; vol.7/Exp. 3/f. 210r.).

7. Las disposiciones de los febricitantes son más a el propósito por el mismo hecho de estar sus cuerpos más calientes (México, 25/02/1793; vol. 7/Exp. 3/f. 209v.).

8. Les hacía mover el vientre con lavativas emolientes que hacía componer de un cocimiento de malvas (Campeche, 19/03/1793; vol. 7/Exp. 8/f. 332r.).

9. Siguiendo los convalecientes este desorden, han recaído y algunos de esta recidiva han fallecido (Zimapán, 06/02/1794; vol. 17/Exp. 1/f. 58v.).

10. Padecen los virulentos convulsiones, delirios, desvelos, dolores en varias partes del cuerpo, hinchazones, intumescencias (...) disenterías, hidropesías parciales y totales (Campeche, 18/03/1793; vol, 7/Exp. 8/f. 334r.).

11. Hay siete hombres y cuatro mujeres en sus respectivas enfermerías, las otros dos, una tiene un tumor cancroso (Zimapán, 06/02/1794; vol. 17/ Exp. 1/f. 58v.).

12. El síntoma que se presentó la noche de ayer (...) fue un escupido cruento, a causa de conato nauseoso (Orizaba, 12 de septiembre de 1797; vol. 5/Exp. 2/f. 67r.).

13. Dilatarían la membrana celular disponiéndola a recibir todo el veneno viroloso contenido en la sangre (Campeche, 19/03/1793; vol. 7/Exp. 8/f. 332r.).

Mención aparte merece, por supuesto, la incorporación en el discurso médico de voces tomadas de las grandes lenguas de la ciencia en la sociedad dieciochesca, el latín y en menor medida el griego: a este respecto, el corpus aquí analizado refleja el uso abundante de vocablos de estos orígenes -tales como caquéctico, disentería, erisipela, hidropesía o metástasis (ejemplos 14-18)-, si bien hay que indicar que se trata, en general, de términos conocidos desde antiguo en español ${ }^{21}$ y con presencia ya en la lexicografía dieciochesca especializada ${ }^{22}$.

21. En concreto, las dataciones de CORDE (REAl ACADEmia Española. Banco de datos CORDE. Corpus diacrónico del español. En red: <http://www.rae.es>, 2014 [Consulta: 11/05/2014]) para tales voces son las siguientes: disentería, 1450-1500; erisipela, 1599; hidropesía, 1481. Más modernas parecen ser caquéctico, que en el corpus de la Academia se registra por primera vez en 1807 -pero que el FGRAE (Real Academia Española. Fichero General de la Real Academia Española. En red: <http:// www.rae.es>, 2014; s.v. caquéctico) descubre con cierta abundancia en obras dieciochescas-, y metástasis, que con su valor médico no aparece hasta un muy tardío 1876, si bien el propio Terreros (Terreros, Esteban de. Diccionario castellano con las voces de las ciencias y las artes. Madrid: ArcoLibros, 1987 [1786-8]; s.v. metástasis) ofrece ya este significado en su diccionario.

22. TERreros, Esteban de. Diccionario castellano con las voces de las ciencias y las artes. Madrid: ArcoLibros, 1987 [1786-8]; s.v. caquéctico, disentería, erisipela, hidropesía, metástasis. 
14. Juan Esteban de 18 años caquéctico venero, padece viruelas bastardas (Ciudad de México, 24/09/1797; vol. 11/Exp. 2/f. 151r.).

15. Padecen los virulentos convulsiones, delirios, desvelos, dolores en varias partes del cuerpo, inchazones, entumescencias (...) disenterías, hidropesías parciales y totales (Campeche, 18/03/1793; vol, 7/Exp. 8/f. 334r.).

16. Se observan en todo el año y en todas sus estaciones sin llegar al grado de epidemia, y a la verdad se equivoca con la erisipela vejigosa (Xalapa, 16/06/1790; vol. 7/Exp. 1/f. 87r.).

17. Padecen los virulentos convulsiones, delirios, desvelos, dolores en varias partes del cuerpo, inchazones, entumescencias (...) disenterías, bidropesías parciales y totales (Campeche, 18/03/1793; vol, 7/Exp. 8/f. 334r.).

18. Se ha advertido en esta jurisdicción unos dolores vagos, epidémicos que el retroceso de ellos o metástasis imperfecto termina en espurios y pleuríticos (Ciudad de México, 24/01/1794; vol. 17/Exp. 1/f. 3r.).

Ahora bien, no todos los latinismos que ofrecen los documentos son, como los anteriores, voces de antigua presencia en el español; muy al contrario, es posible señalar también el empleo completamente integrado -esto es, como voces consideradas romances- de otros que, si bien aparecen en la bibliografía médica española y europea del Siglo de las Luces, en tales obras -y en claro contraste con lo que ocurre en esta documentación-, mantienen siempre su carácter latino, al aparecer en textos escritos en esta lengua o bien como voz propia de este idioma. Tal es el caso, por ejemplo, de variola, frecuentemente utilizado en el corpus como sinónimo del romance viruela (ejemplo 19), o de maleficencia 'enfermedad' (ejemplos 20, 21), que se incorpora en 1803 al DRAE ${ }^{23}$ exclusivamente con su valor de 'hábito o costumbre de hacer el mal'24, pero cuyo empleo latino con el significado médico ya señalado es posible descubrir en obras especializadas del siglo XVII, tales como Dureto ${ }^{25}$ o Schneider $^{26}$, entre otros muchos ${ }^{27}$.

23. Real Academia Española. Diccionario de la lengua castellana. 4. a edición. Madrid: Viuda de Ibarra, 1803; s.v. maleficencia.

24. Esta incorporación en los primeros años del siglo XIX casa con la opinión de Hildebrandt (Hildebrandt, Martha. La lengua de Bolivar, I. Léxico. Caracas: Universidad Central de Venezuela, 1961: p. 356), quien lo considera uno de los «latinismos que eran neológicos cuando Bolívar los usó, es decir, los que se incorporaron a la lengua española durante el siglo XIX"; sin embargo, hay que indicar que su uso con el significado recogido por la Academia no era desconocido en el siglo XVII, según evidencian entre otros Tesauro (TESAUro, Emmanuele. Filosofía moral. Lisboa: Antonio Craesbeeck, 1682; pp. 113, 238) y Solares (SOLARES, Francisco. El emperador politico y política de emperadores, II. Barcelona: Joseph Llopis, 1700; p. 222).

25. Dureto, Ludovico. Hippocrates magni coacae praenotationes. Ginebra: Gamonet, 1665.

26. SCHENEIDER, Konrad Victor. Liber de morbis capitis. Wittenberg: M. Henckell, 1669.

27. Por lo que se refiere a la bibliografía española, esta última voz se emplea, por ejemplo, en el tratado médico de Rubio (RubIo, Francisco. Medicina Hippocratica ó Arte de conocer y curar las 
19. Tiene la variola maligna, toda sembrada de petequias negras (Orizaba, ?/05/1797; vol. 5/Exp. 2/f. 169r.).

20. Su materia corrosiva había ulcerado las vísceras nobles interesando su maleficencia el diafragma y pulmón (Chalco, 01/04/1797; vol. 3/Exp.2/f. 88v.).

21. Hasta ahora, Dios mediante, no se experimenta aquí semejante maleficencia, ni hay la más mínima prelución de tal contagio (Chilapam, 16/08/1796; vol. 3/Exp. 1/f. 46v.).

3.2. Frente a todo lo anterior, sin duda resultan de mayor interés en el corpus las ya mencionadas "voces de los pacientes" ${ }^{28}$, es decir, aquellas denominaciones populares con que los no especialistas se refieren a la enfermedad y a sus consecuencias; a este respecto, cabe recordar una vez más que la obsesión burocrática hispánica de dejar constancia por escrito de todos los aspectos de la vida permite acceder por medio de la documentación de archivo «a ámbitos de uso -y, por tanto, a unidades léxicas- que no aparecen en otros tipos textuales ${ }^{29}$, y que resultan interesantes también a la hora de desarrollar un análisis más completo del vocabulario propio de la medicina.

Por supuesto, no resulta sencillo definir qué se entiende por voces populares, denominación claramente ambigua que es necesario precisar más allá de lo indicado en el párrafo anterior ${ }^{30}$; afortunadamente, en algunas ocasiones son los mismos textos que componen el corpus o diversos trabajos médicos de la época

enfermedades por reglas. Madrid: Imprenta Real de la Gaceta; p. 266), pero no con el significado de 'enfermedad', según se descubre en la siguiente cita: «si se altera en alguna constitución aquel espíritu o causa general de manera que decae su beneficencia o se exaspera su maleficencia se notan al instante en los males estas mudanzas al modo que se resiente». En cuanto a variola, se descubre ya como voz latina en obras médicas del siglo XV, y aparece en el DRAE en 1817 (REAL ACAdemia EsPaÑola. Diccionario de la lengua castellana. $5^{\text {a }}$ edición. Madrid: Real Academia Española, 1817; s.v. viruela), pero como mera traducción de la española viruela (RAMírez LuENGO, José Luis. "Un aporte a la historia del léxico médico en América: el vocabulario de la viruela en la Nueva España dieciochesca». Anuario de Letras. Lingüística y Filología, 2014, II/1 (en prensa).

28. GÓMEZ DE ENTERRÍA, Josefa y Natividad GALLARDO. "Las versiones de medicina y botánica y la nueva terminología científica en el siglo XVIII. Cuadernos del Instituto de Historia de la Lengua, 2010, 4, pp. 55-75; p. 58.

29. RAmírez Luengo, José Luis. "Un aporte a la historia del léxico médico en América: el vocabulario de la viruela en la Nueva España dieciochesca». Anuario de Letras. Lingüística y Filología, 2014, II/1 (en prensa).

30. Aunque pueda resultar más que evidente, se hace necesario indicar que en la valoración de un elemento como voz popular se atenderá exclusivamente a cuestiones de carácter léxico -idealmente, ámbitos de uso y grupos sociales que la utilizan- y no a aspectos de otro tipo: por ejemplo, no se dará tal consideración a los términos del corpus caquético, entumescencia, malificencia o metástesis por más que en ellos aparezcan fenómenos fónicos propios hoy de los diastratos populares como son la simplificación de los grupos cultos o la vacilación en las vocales átonas (LAPESA, Rafael. Historia de la lengua española. $9^{a}$ edición. Madrid: Gredos, 1981; pp. 368, 421). 
los que facilitan la valoración que se debe conceder a determinadas unidades léxicas, al indicar de variadas formas - pero con una clara preferencia por la alusión al vulgo-, su carácter de voces populares: tal es el caso, por ejemplo, de la voz engrasado -referida al paciente de cierta enfermedad- (ejemplo 22) y del sintagma ampollas de Naolingo 'mancha en la piel parecida a la que produce la viruela' (ejemplo 23), que no se recogen en ningún corpus o fuente lexicográfica consultados y, que -de acuerdo con sus contextos de aparición en el corpus- parece ser términos propios del registro no especializado y, además, de carácter geográficamente muy restringido ${ }^{31}$.

22. Los he visitado y encontrado a más de la enfermedad epidémica varios pletícicos (?) vulgarmente nombrados en este lugar engrasados (Zimapan, 06/02/1791; vol. 17/Exp. 1/f. 59r.).

23. Observamos también algunas pintas que imitan a las que dejan la viruela (...) las que el vulgo aquí llama ampollas de naolingo (Xalapa, 16/06/1790; vol. 7/Exp. 1/f. 87r.).

Algo semejante ocurre con los más generales pinta, tabardillo o vómito prieto, cuya aparición en obras médicas de la época suele venir acompañada de la ya mencionada alusión a su carácter popular, por más que en el corpus manejado para este estudio no se indique tal circunstancia (ejemplos 24-26): de nuevo con una alusión a su vulgaridad son calificadas las dos últimas voces en los títulos de las obras que $\operatorname{Amar}^{32}$ y Salvá y Campillo ${ }^{33}$ dedican a estas dolencias, denominadas Instrucción curativa de las calenturas conocidas vulgarmente con el nombre de tabardillo y Análisis de la fiebre llamada vulgar e impropiamente amarilla, o vómito prieto respectivamente ${ }^{34}$, y tal calificación se repite en el caso de pinta

31. En el caso concreto del segundo elemento, probablemente de la zona del Golfo de México -actual estado de Veracruz-, habida cuenta de cuestiones como la indicación de los autores del texto de que "el vulgo aquí llama», el hecho de que ese aquí se pueda identificar con la ciudad de Xalapa, lugar donde se data el documento, y muy especialmente el empleo en el sintagma del topónimo Naolingo, denominación habitual en el siglo XVIII del municipio veracruzano de Naolinco.

32. AMAR, José. Instrucción curativa de las calenturas conocidas vulgarmente con el nombre de tabardillo. Madrid: Joaquín Ibarra, 1775.

33. Salvá y CAMPILLO, Francisco. Análisis de la fiebre llamada vulgar e impropiamente amarilla, o vómito prieto. Barcelona: Viuda de A. Brusi e hijos, 1821.

34. Por lo que se refiere al tabardillo, el carácter popular de la denominación queda claramente explicitado por Virrey y Mange (VIRREY y MANGE, Pascual F. Palma febril médico-práctica, II. Madrid: Antonio Sanz, 1756; p. 113) al indicar que «llámase últimamente esta fiebre tabardillo nombre muy usado en el vulgo castellano y aragonés», para el cual, además, aporta una explicación etimológica: "quieren derivar este nombre de un animalillo muy pintado llamado tabardo y de aquí originan al tabardillo"; por si fuera poco, una búsqueda en CORDE (REAL ACADEMIA ESPAÑOLA. Banco de datos CORDE. Corpus diacrónico del español. En red: <http://www.rae.es>, 2014 [Consulta: 12/05/2014]) ofrece un total de 70 apariciones del vocablo entre 1650 y 1850, de las que únicamente dos -es decir, el 2'85\%se registran en textos médicos o relacionados con la medicina. En cuanto al vómito prieto, quizá sea 
'mancha en la piel', según refleja, por ejemplo, la definición de Juan de Vidos y Miró $^{35}$, quien habla de «algunas manchas dichas vulgarmente pecas o pintas».

24. En el segundo tiempo que es cuando aparece la erupción, que se conocía por las pintas rojas que aparecían a manera de picadas de pulgas (Campeche, 19/03/1793; vol. 7/Exp. 8/f. 332v.).

25. He suspendido la formación de padrones anuales (...) a causa de la vigorosa peste de viruelas, tabardillos y otros síntomas que han cundido en esta Jurisdicción (s.1., 31/12/1779; Ind. Virreinal/Caja 1807/Exp. 22/f. 1r.).

26. En la semana anterior ha fallecido en esta parroquia de Sn. Antonio seis enfermos de viruelas naturales, y uno de vómito prieto (Ciudad de México, 5/11/1797; vol.1/Exp. 1/f. 112r.).

Esta incorporación de voces populares al léxico técnico especializado que dejan ver las citas anteriormente expuestas obliga a considerar que la dirección de la expansión en el uso de estos elementos - al menos en la época analizada- no va exclusivamente desde el ámbito científico hacia lo popular, sino también a la inversa; es decir, que en su estudio el investigador se va a enfrentar a "voces de la medicina que trascienden el nivel de los especialistas y llegan hasta el enfermo ${ }^{36}$,

interesante señalar no solo el carácter popular de la denominación, sino también -y muy especialmente- la relación que esta voz parece tener con el español americano: en este sentido, Aréjula (ARÉJUla, Juan Manuel de. Breve descripción de la fiebre amarilla padecida en Cádiz y pueblos comarcanos en 1800. Madrid: Imprenta Real, 1806; p. 147) señala que «los españoles han llamado y nombran vómito prieto en las Américas Septentrionales a una calentura pútrida no contagiosa", mientras que, al hablar de la piña, el Diccionario de Autoridades (REAl ACADEMIA ESPAÑOla. Diccionario de Autoridades (edición facsímil de la de 1726-1739). Madrid: Gredos, 1979; s.v. piña) dice que "en las partes ardientes de la América la toman como preservativo para el vómito prietom; súmese a esto que los únicos registros que se localizan en CORDE (REAL ACADEMIA ESPAÑOLA. Banco de datos CORDE. Corpus diacrónico del español. En red: <http://www.rae.es>, 2014 [Consulta: 11/05/2014]) en el periodo comprendido entre 1650 y 1850 corresponden a Ulloa en su Viaje al Reino del Perú y a José Celestino Mutis en su epistolario. Ambas circunstancias -su carácter popular y su especial relación con el español americano y/o mexicano- parecen condensarse en la cita de Lara (LARA, Luis Fernando. (dir.). Diccionario del español de México. México D. F.: El Colegio de México, 2010; s.v. vómito), quien, al hablar del vómito negro, indica que "coloquialmente se usa a veces vómito prieto». Parece bastante probable, por tanto, que -al igual que pasaba con varioloso y viruelento (RAmírez LuENGO, José Luis. "Un aporte a la historia del léxico médico en América: el vocabulario de la viruela en la Nueva España dieciochesca". Anuario de Letras. Lingüística y Filología, II/1, 2014; en prensa)- este elemento se pueda considerar un americanismo en el léxico de la medicina, si bien es aún necesario llevar a cabo estudios más profundos que confirmen esta hipótesis.

35. VIDOS y MiRó, Juan de. Medicina y cirugía racional y espagirica, II. Zaragoza: Pascual Bueno, 1720; p. 18.

36. Gómez de Enterría, Josefa; NAvarro, Carmen; Dalle Pezze, Francesca y Elena Carpi. "Los documentos como fuente de investigación lexicológica y lexicográfica en corpus de textos de especialidad del siglo XVIII». En Torrens Álvarez, María Jesús y SÁnchez-Prieto Borja, Pedro (eds.). Nuevas 
pero también al proceso contrario, de vocablos populares que terminan por incorporarse al discurso especializado ${ }^{37}$. A partir de esta constatación, es posible extraer dos ideas de cierto interés: por un lado, que son muchas las dificultades que conlleva intentar establecer la dirección de la expansión en el uso en casos como, por ejemplo, comezón o calentura, ambos presentes en el corpus (Campeche, 19/03/1793, en AGN Epidemias (044), vol. 7/Exp.8/f. 333r. y Veracruz, 01/03/1809, en AGN Epidemias (044), vol. 17/Exp. 7/f. 184r., respectivamente) y frecuentes durante el siglo XVIII tanto en obras médicas como en otras ajenas a este campo ${ }^{38}$; por otro $-\mathrm{y}$ desde un punto de vista metodológico-, la imposibilidad de considerar la presencia/ausencia de estos términos en el discurso especializado como índice de su carácter culto o popular, habida cuenta de que, como se acaba de decir, no resulta extraño que voces de origen claramente popular -y consideradas de tal modo por los profesionales de la época, a la luz de su definición como vulgares- se incorporen y aparezcan empleadas en este discurso.

Por otro lado, cabe señalar la presencia en los documentos de algunas voces que muy probablemente pertenecen también al ámbito del vocabulario popular que se está analizando en este apartado, pero para cuya valoración no se cuenta con indicaciones incuestionables como las señaladas más arriba, sino con diferentes indicios que es necesario interpretar desde muy diferentes puntos de vista. Así, es posible que como voz popular se deba interpretar el indigenismo matlazahua (ejemplo 27), que hace su presencia en los documentos con cierta asiduidad para referirse a una «fiebre eruptiva que cubre el cuerpo de manchas

perspectivas para la edición y el estudio de documentos hispánicos antiguos. Berna: Peter Lang, 2012, pp. 127-146; p. 133.

37. Así, se constata que, en el caso concreto del léxico médico, no siempre es aplicable la jerarquía que establece Lopez (LOPEz, François. Juan Pablo Forner et la crise de la conscience espagnole au XVIIIè siècle. Burdeos: Universidad de Burdeos, 1976; p. 61) para medir el grado de penetración de las voces que se están analizando: en efecto, si para este autor tal penetración va desde los «traites scientifiques" a lo literario y de ahí a "le langage écrit le plus courant", estos ejemplos aquí mencionados parecen suponer un proceso de penetración inverso al mencionado, e incluso es muy probable que en ocasiones se pueda descubrir en el primer y tercer grado de penetración y no en el segundo, todo lo cual no hace sino aumentar las dificultades que señalaba ya al respecto Álvarez de Miranda (Álvarez de Miranda, Pedro. Palabras e ideas. El léxico de la Ilustración temprana en España. Madrid: Real Academia Española, 1992; pp. 70-1) y dibuja, al mismo tiempo, una situación mucho más flexible y dinámica en lo que tiene que ver con la incorporación y generalización de las diferentes unidades léxicas en la lengua.

38. Real Academia Española. Banco de datos CORDE. Corpus diacrónico del español. En red: <http://www.rae.es>, 2014 (Consulta: 11/05/2014). Por lo que se refiere a esta última, el DCECH (COROminas, Joan y José Antonio PASCuAl. Diccionario crítico etimológico castellano e hispánico. Madrid: Gredos, 1980-1991; s.v. fiebre) parece avalar su carácter popular al indicar que, frente a fiebre, "la denominación realmente popular fue siempre calentura", que data, además, ya en 1340 (COROMINAS, Joan y José Antonio Pascual. Diccionario crítico etimológico castellano e hispánico. Madrid: Gredos, 1980-1991; s.v. calor); por supuesto, ambas voces -calentura y comezón- son de uso común hoy en día en México (LARA, Luis Fernando. (dir.). Diccionario del español de México. México D. F.: El Colegio de México, 2010; s.v. calentura, comezón). 
rojas y que padecían los antiguos mejicanos ${ }^{39}$ y que genera, de acuerdo con Molina del Villar ${ }^{40}$, una de las epidemias más desastrosas del siglo XVIII en el actual México $^{41}$; a este respecto, el hecho de que el origen de la voz se encuentre en una de las lenguas originarias de la región, el náhuatl, obliga a pensar en un primer empleo entre los estratos más populares de la población, a partir del cual pasa a documentos notariales como los que se analizan en estos momentos y también a textos que, en cierto modo, se pueden considerar representativos del discurso especializado, al estilo, por ejemplo, del que publica Cabrera y Quintero ${ }^{42}$ en $1746^{43}$.

27. Encontré dos pestes distintas pues hay muchos virulentos, y algunos con fiebres pútridas petequiados que aquí llaman matlazagua (Tacuba, 05/09/1797; vol. 11/Exp. 2/f. 72r.).

En esta misma línea, resulta interesante también analizar las voces pegoso 'contagioso' y enronchado 'cubierto de ronchas' ${ }^{\prime 4}$, no solo por su indudable carácter popular, sino también por su relación con el español americano. Por lo que se refiere a pegoso (ejemplo 28), cabe decir que no aparece en ninguno de los repertorios lexicográficos consultados ni tampoco en los grandes repositorios de la Real Academia ${ }^{45}$ con la significación que ofrece el texto ${ }^{46}$, de manera que por el momento constituye un hápax cuya extensión e historia será necesario analizar

39. Santamaría, Francisco. Diccionario de Mejicanismos. México DF.: Porrúa, 1992; s.v. matlazagua.

40. Molina del Villar, América. Remedios contra la enfermedad y el hambre. En GonZalbo AizPuru, Pilar (coord). Historia de la vida cotidiana en México, III. México DF.: El Colegio de México - Fondo de Cultura Económica, 2005, pp. 179-212; p. 170.

41. Para una descripción histórica de la dolencia y de la gran epidemia de los años treinta del siglo XVIII, véase Molina del Villar (Molina Del Villar, América. La Nueva España y el matlazahuatl, 1736-1739. México DF.: Centro de Investigaciones y Estudios Superiores en Antropología Social - El Colegio de Michoacán, 2001), así como Cuenya (CuenYA, Miguel Ángel. Peste en una ciudad novohispana: el matlazáhuatl de 1737 en la Puebla de los Ángeles. Anuario de Estudios Americanos, 53/2, 1996, pp. 51-70), quien centra el estudio en el caso concreto de la ciudad de Puebla.

42. Cabrera y Quintero, Cayetano. Escudo de armas de México. México: Viuda de D. Joseph Bernardo de Hogal, 1746

43. Algo semejante se puede señalar para el quechuismo quina (Campeche, 19/03/1793; vol. 7/Exp.8/f. 333v.), que se incorpora al español durante los primeros años del siglo XVII y resulta de uso muy frecuente en textos científicos de diversas zonas del mundo hispánico durante la centuria siguiente (Real ACADEmia Española. Banco de datos CORDE. Corpus diacrónico del español. En red: <http://www.rae.es>, 2014 [Consulta: 16/05/2014]), en consonancia con los documentos novohispanos aquí analizados.

44. Real ACAdemia Española. Diccionario de la Lengua Española. 22a edición. Madrid: EspasaCalpe, 2001; s.v. enronchar.

45. Real Academia Española. Banco de datos CORDE. Corpus diacrónico del español. En red: <http://www.rae.es>, 2014; ReAl ACADEMIA EsPañola. Banco de datos CREA. Corpus de referencia del español actual. En red: <http://www.rae.es>, 2014 (Consulta: 16/05/2014).

46. Aparece, eso sí, en el FGRAE (REAL ACADEMIa EsPañola. Fichero General de la Real Academia Española. En red: <http://www.rae.es>, 2014; s.v. pegoso) como vocablo andaluz con el valor de 
en el futuro a la luz de nueva documentación ${ }^{47}$; con todo, su acuñación popular no resulta difícil de comprender si se tiene en cuenta, por un lado, el empleo de pegar con el significado de 'contagiar'48, y por otro, la manifiesta preferencia -al menos actual- del español mexicano por el sufijo -oso para formar adjetivos que expresan cualidad, circunstancias a partir de las cuales la aparición del término con el significado señalado se encuentra sin duda dentro de lo esperable ${ }^{49}$.

28. Su Superioridad me dice que con el mayor cuidado y esmero proporcione paraje donde puedan ponerse los epidémicos de viruelas, por ser esta enfermedad muy pegosa (Pachuca, 08/04/1797; vol. 3/Exp. 2/f. 81r.).

En cuanto al sustantivo enronchado (ejemplo 29), una vez más se trata de un término con escasa documentación lexicográfica, y caracterizado en todas las ocasiones por su relación con América: así, el verbo enronchar aparece por primera vez en el diccionario de Alemany ${ }^{50}$ con la marca diatópica de Chile, algo que se mantiene sin cambios $-y$, de acuerdo con estos datos, impropiamenteen el DRAE ${ }^{51}$; a esto se ha de sumar, además, su presencia en el diccionario de

'persona que dice tonterías', pero es evidente que nada tiene que ver este término con el analizado en estas páginas.

47. A este respecto, cabe mencionar que tampoco se registra en los diccionarios del español actual de México de Santamaría (Santamaría, Francisco. Diccionario de Mejicanismos. México D. F.: Porrúa, 1992) y Lara (LARA, Luis Fernando. (dir.). Diccionario del español de México. México D. F.: El Colegio de México, 2010).

48. Real Academia Española. Diccionario de la Lengua Española. 22. a edición. Madrid: EspasaCalpe, 2001; s.v. pegar. Valor, por cierto, presente ya en el siglo XVIII, como atestigua Autoridades (Real Academia Española. Diccionario de Autoridades (edición facsímil de la de 1726-1739). Madrid: Gredos, 1979; s.v. pegar): "vale también comunicar una cosa a otra. Comúnmente se dice de las enfermedades contagiosas".

49. En esta misma línea, es necesario señalar también la voz tabardillento (vol. 17/Exp. 3/f. $141 \mathrm{v}$.), que, al igual que en el caso de pegoso, constituye un hápax de la documentación aquí analizada: "En el Hospital de San Andrés tal vez aborda, si no pasa, el número de setecientos enfermos, siendo proporcionalmente considerable el de los tabardillentos" (Ciudad de México, 01/02/1803). Una vez más, es posible que se deba interpretar como una voz popular - de ahí su nula documentación diacrónica o sincrónica- construida por analogía con otras como calenturiento o virolento para referirse al enfermo que padece la enfermedad en cuestión; queda ahora por analizar, por supuesto, si se trata de una voz efímera (GÓMEZ DE ENTERRÍA, Josefa. "El vocabulario de las fiebres epidémicas en español del siglo XVIII». En: Ramírez Luengo, José Luis y Velásquez Upegui, Eva Patricia (eds.). La historia del español hoy. Estudios y perspectivas. Lugo: Axac; en prensa) pero de uso en el español -siquiera novohispano- de la Centuria Ilustrada o de una mera acuñación puntual que no transciende el informe administrativo donde se registra -hasta el momento- por primera y única vez.

50. Alemany, José. Diccionario de la lengua española. Barcelona: Ramón Sopena, 1917; s.v. enronchar.

51. Real Academia Española. Diccionario de la Lengua Española. 22. a edición. Madrid: EspasaCalpe, 2001; s.v. enronchar. De forma paradójica - pero en clara relación con lo que se dirá inmediatamente-, la primera datación de este elemento en CORDE (REAL ACADEMIA EsPañola. Banco de datos CORDE. Corpus diacrónico del español. En red: <http://www.rae.es>, 2014 [Consulta: 16/05/2014]) no se documenta en Chile, sino en un texto argentino decimonónico, en concreto en el Informe del 
mexicanismos de Santamaría ${ }^{52}$ y el hecho de que los únicos ejemplos registrados en CREA $^{53}$ se localicen en Nicaragua y Cuba, lo que -si bien obliga a matizar la distribución geográfica señalada en el DRAE- avala el uso americano de la voz, algo que también señala el FGRAE ${ }^{54}$ al indicar que en España resulta «menos frecuente» que en el Nuevo Mundo.

29. Todavía no hay sarpuchidos (sic) ni enronchados, por lo que no hay contagio en el día (s.1., 1797?; vol. 5/Exp. 3/f. 223r.).

Se trata, por tanto, de dos voces que parecen guardar una estrecha relación con el español americano y que -al igual que se postulaba para varioloso y viruelento ${ }^{55}$ y quizá para vómito prieto- es posible entender como americanismos: así, teniendo en cuenta los subtipos que engloba tal concepto y siguiendo, una vez más, la clasificación que establece al respecto Company ${ }^{56}$, enronchar probablemente pertenezca a los americanismos de frecuencia, al igual que vómito prieto, mientras que pegoso 'contagioso' -así como el indigenismo matlazahua y quizá engrasado, ampolla de naolingo y tabardillento- se deben incluir dentro de los americanismos puros. En todo caso, y más allá de la clasificación concreta, lo que se pretende resaltar aquí es el hecho de que efectivamente existen voces relacionadas con la medicina que están determinadas geográficamente, y que tales voces, como se puede ver por este análisis -y como resulta, por otro lado, más que esperable- son especialmente abundantes en el léxico que, de manera más o menos imprecisa, se ha denominado popular.

4. De este modo, el estudio llevado a cabo a lo largo de estas páginas permite poner de manifiesto una vez más la importancia que posee la documentación notarial a la hora de llevar a cabo un análisis diacrónico más profundo del léxico de la medicina, no solo por la gran abundancia de términos relativos a la materia que se descubre en estos textos, sino también -y muy especialmente- porque la redacción de tales documentos por parte de autores muy variados -muchos de los cuales no se pueden considerar de ningún modo especialistas en la práctica médica- permite la aparición de un importante número de unidades léxicas que

Departamento Nacional de Agricultura (Buenos Aires?: Imprenta del Porvenir, 1875; p. 409): «he visto un hombre que, de rozar un palo casi seco, se ha enronchadon.

52. Santamaría, Francisco. Diccionario de Mejicanismos. México D. F.: Porrúa, 1992; s.v. enronchar.

53. Real ACAdemia Española. Banco de datos CREA. Corpus de referencia del español actual. En red: <http://www.rae.es>, 2014 (Comsulta: 16/05/2014).

54. Real Academia Española. Fichero General de la Real Academia Española. En red: <http:// Www.rae.es>, 2014; s.v. enronchar.

55. RAmírez Luengo, José Luis. "Un aporte a la historia del léxico médico en América: el vocabulario de la viruela en la Nueva España dieciochesca». Anuario de Letras. Lingüística y Filología, 2014, II/1 (en prensa).

56. Company, Concepción. El siglo XVIII y la identidad lingüística de México. México D. F.: Universidad Nacional Autónoma de México - Academia Mexicana de la Lengua, 2007. 
se pueden considerar -de manera quizá poco específica-populares, y que, precisamente por este carácter popular, no suelen aparecer en otros materiales (como tratados científicos u obras de divulgación) que se han empleado habitualmente como base para este tipo de estudios.

Por supuesto, el corpus aquí analizado ofrece un gran número de términos pertenecientes a la esfera del discurso médico establecido, y en este sentido es de destacar que la documentación americana registra las mismas tendencias señaladas por Gómez de Enterría ${ }^{57}$ para los textos españoles de la época, tanto en lo que tiene que ver con las voces empleadas como con los procedimientos neológicos de creación de nuevos elementos léxicos; a este respecto, cabe señalar el empleo de voces especializadas tomadas de diversas lenguas europeas (petequia, pleuresía) y muy especialmente de las lenguas clásicas (caquéctico, disentería, erisipela, hidropesía o metástasis), así como el empleo de ciertos sufijos de manera predominante, entre los que sin duda destacan -nte y -oso.

En cuanto al vocabulario que se ha definido como popular, se hace necesario señalar en primer lugar lo difícil que resulta dar una definición exacta de estos elementos que vaya más allá y sea más precisa que 'denominaciones con que los no especialistas se refieren a la enfermedad y a sus consecuencias'; así, la idea primitiva de que se consideren de este modo todos aquellos elementos que no aparezcan en el ya mencionado discurso médico especializado no resulta del todo válida al constatarse que en numerosas ocasiones las voces populares se incorporan también a este discurso, si bien es cierto que su incorporación suele acompañarse -en este corpus o en otras obras- de cierta referencia al vulgo, tal cual ocurre con tabardillo, vómito prieto, engrasado o ampolla de naolingo. Este hecho, por otro lado, pone de manifiesto que el proceso de expansión de los vocablos en el caso de la medicina no se produce exclusivamente desde el ámbito científico hacia lo popular, sino que en ocasiones tiene lugar de modo contrario, esto es, desde lo popular hacia el discurso especializado, algo que determina no solo una situación mucho más flexible y dinámica al respecto que la establecida por Lopez ${ }^{58}$ en su jerarquía para la medida del grado de penetración de las unidades léxicas, sino que, además, puede dificultar en mucho el análisis de los procesos de expansión de términos como, por ejemplo, comezón o calentura.

57. Gómez de ENTERría, Josefa. "El vocabulario médico de los novatores en el siglo XVIII». En García Godoy, María Teresa (ed.). El español del siglo XVIII. Cambios diacrónicos en el primer español moderno. Berna: Peter Lang, 2012, pp. 55-81; GómEZ DE ENTERRÍA, Josefa. «El vocabulario de las fiebres epidémicas en español del siglo XVIII». En Ramírez Luengo, José Luis y VeLÁsQuez Upegui, Eva Patricia (eds.). La historia del español hoy. Estudios y perspectivas. Lugo: Axac (en prensa).

58. LOPEZ, François. Juan Pablo Forner et la crise de la conscience espagnole au XVIIIè siècle. Burdeos: Universidad de Burdeos, 1976; p. 61. 
Por otro lado, si bien acierta totalmente Gómez de Enterría ${ }^{59}$ cuando indica que no existen diferencias sustanciales entre el léxico médico de España y América en el siglo XVIII más allá de la presencia de algunos indigenismos, lo cierto es que tal aserto resulta válido para el vocabulario culto, pero se debe matizar en el caso de aquel que se puede englobar dentro de lo popular, pues en este caso sí es posible detectar la aparición de una serie de voces diatópicamente marcadas: indigenismos como matlazahua, pero también compuestos del estilo de vómito prieto y voces derivadas como pegoso, enronchar o tabardillento, que se pueden interpretar sin muchas dificultades como americanismos dentro del ámbito especializado de la medicina. Por supuesto, en nada puede sorprender que, junto a un léxico especializado común, se registren también especificidades geográficas que se engloban primordialmente dentro de los vocablos propios de los grupos populares, pues es sin duda esta convivencia de léxico culto y otro más apegado a la realidad más cercana -y, por tanto, geográficamente más diverso- la estrategia fundamental de la que dispone el no especialista a la hora de referirse a una cuestión tan técnica, pero a la vez tan cotidiana, como es, a lo largo de la historia, la enfermedad y sus consecuencias.

\section{BIBLIOGRAFÍA CITADA}

Alemany, José. Diccionario de la lengua española. Barcelona: Ramón Sopena, 1917.

Álvarez de Miranda, Pedro. Palabras e ideas. El léxico de la Ilustración temprana en España. Madrid: Real Academia Española, 1992.

Álvarez De Miranda, Pedro. "El léxico español, desde el siglo XVIII hasta hoy». En Cano, Rafael (coord.). Historia de la lengua española. Barcelona: Ariel, 2004, pp. 1037-1064.

AmAR, José. Instrucción curativa de las calenturas conocidas vulgarmente con el nombre de tabardillo. Madrid: Joaquín Ibarra, 1775.

ARÉJula, Juan Manuel de. Breve descripción de la fiebre amarilla padecida en Cádiz y pueblos comarcanos en 1800. Madrid: Imprenta Real, 1806.

Cabrera y Quintero, Cayetano. Escudo de armas de México. México: Viuda de D. Joseph Bernardo de Hogal, 1746.

Company, Concepción. El siglo XVIII y la identidad lingüística de México. México D. F.: Universidad Nacional Autónoma de México - Academia Mexicana de la Lengua, 2007.

Corde. Real academia Española. Banco de datos CORDE. Corpus diacrónico del español. En red: <http://www.rae.es>, 2014.

CREa. Real Academia Española. Banco de datos CREA. Corpus de referencia del español actual. En red: <http://www.rae.es>, 2014.

CuenYa, Miguel Ángel. «Peste en una ciudad novohispana: el matlazáhuatl de 1737 en la Puebla de los Ángeles». Anuario de Estudios Americanos, 1996, 53/2, pp. 51-70.

59. GÓmEZ DE ENTERRía, Josefa. «El vocabulario de las fiebres epidémicas en español del siglo XVIII». En Ramírez Luengo, José Luis y Velásquez Upegui, Eva Patricia (eds.). La historia del español boy. Estudios y perspectivas. Lugo: Axac (en prensa). 
DCECH. COROminas, Joan y José Antonio Pascual. Diccionario crítico etimológico castellano e hispánico. Madrid: Gredos, 1980-1991.

Drae, 1803. Real Academia Española. Diccionario de la lengua castellana. 4. ${ }^{a}$ edición. Madrid: Viuda de Ibarra, 1803.

DRAE, 1817. Real ACADEmia Española. Diccionario de la lengua castellana. 5. a edición. Madrid: Real Academia Española, 1817.

Drae, 2001. Real Academia Española. Diccionario de la Lengua Española. 22. a edición. Madrid: Espasa-Calpe, 1992.

Dureto, Ludovico. Hippocrates magni coacae praenotationes. Ginebra: Gamonet, 1665.

FGRAE. Real ACADEmia Española. Fichero General de la Real Academia Española. En red: $<$ http://www.rae.es>, 2014.

GÓMEZ DE ENTERRÍA, Josefa. «El vocabulario médico de los novatores en el siglo XVIII». En García Godoy, María Teresa (ed.). El español del siglo XVIII. Cambios diacrónicos en el primer español moderno. Berna: Peter Lang, 2012, pp. 55-81.

GÓMEZ DE ENTERRíA, Josefa. «El vocabulario de la medicina del siglo XVIII a través de las traducciones de los hermanos Juan y Félix Galisteo Xiorro». En Montero Cartelle (ed.). Actas del VIII Congreso Internacional de Historia de la Lengua Española, II. Santiago de Compostela: Meubook, 2012b, pp. 1405-1422.

GÓMEZ DE ENTERRÍA, Josefa. "Higiene y salud en las traducciones médicas del francés al español durante el siglo XVIII..Panace@. Revista de medicina, lenguaje y traducción, 2013, 14/38, pp. 287-295.

GÓMEZ DE ENTERRÍA, Josefa. «El vocabulario de las fiebres epidémicas en español del siglo XVIII». En Ramírez Luengo, José luis y Velásquez Upegui, Eva Patricia (eds.). La historia del español hoy. Estudios y perspectivas. Lugo: Axac (en prensa).

GÓMEZ DE ENTERRía, Josefa y GALLARDO, Natividad. "Las versiones de medicina y botánica y la nueva terminología científica en el siglo XVIII". Cuadernos del Instituto de Historia de la Lengua, 2010, 4, pp. 55-75.

Gómez de Enterría, Josefa; Navarro, Carmen; Dalle Pezze, Francesca y CarPi, Elena. «Los documentos como fuente de investigación lexicológica y lexicográfica en corpus de textos de especialidad del siglo XVIII». En Torrens Álvarez, María Jesús y SánchezPRIETO Borja, Pedro (eds.). Nuevas perspectivas para la edición y el estudio de documentos hispánicos antiguos. Berna: Peter Lang, 2012, pp. 127-146.

GuTIÉRREZ Rodilla, Bertha M. "Los términos relacionados con la medicina en el Diccionario de Autoridades». Boletín de la Real Academia Española, 1993, 73/260, pp. 463-512.

GuTIÉRREZ RoDilla, Bertha M. "Construcción y fuentes utilizadas para los términos médicos en el Diccionario de Autoridades». Revista de Lexicografia, 1994-5, 1, pp. 149-162.

GuTiÉRreZ Rodilla, Bertha M. «El léxico de la medicina en el diccionario de Esteban Terreros y Pandom. En Alonso GonZÁlez, Alegría et al. (eds.). Actas del III Congreso Internacional de Historia de la Lengua Española, II. Madrid: ArcoLibros, 1996, pp. 1327-1342.

Hildebrandt, Martha. La lengua de Bolivar, I. Léxico. Caracas: Universidad Central de Venezuela, 1961.

LAPESA, Rafael. Historia de la lengua española. 9. a edición. Madrid: Gredos, 1981.

Lara, Luis Fernando. (dir.). Diccionario del español de México. México D. F.: El Colegio de México, 2010.

Lopez, François. Juan Pablo Forner et la crise de la conscience espagnole au XVIIIè siècle. Burdeos: Universidad de Burdeos, 1976. 
Molina Del Villar, América. La Nueva España y el matlazahuatl, 1736-1739. México D. F.: Centro de Investigaciones y Estudios Superiores en Antropología Social y El Colegio de Michoacán, 2001.

Molina Del Villar, América. "Remedios contra la enfermedad y el hambre». En GonzalBo Aizpuru, Pilar (coord.). Historia de la vida cotidiana en México, III. México D. F.: El Colegio de México y Fondo de Cultura Económica, 2005, pp. 179-212.

Ramírez Luengo, José Luis. "Aproximación al léxico de la medicina en el Buenos Aires del siglo XVIII». En Actas del IX Congreso Internacional de Historia de la Lengua Española (en prensa).

Ramírez Luengo, José Luis. "Un aporte a la historia del léxico médico en América: el vocabulario de la viruela en la Nueva España dieciochesca». Anuario de Letras. Lingüística y Filología, 2014, II/1 (en prensa b).

Real Academia Española. Diccionario de Autoridades (edición facsímil de la de 1726-1739). Madrid: Gredos, 1979.

RuBIO, Francisco. Medicina Hippocratica ó Arte de conocer y curar las enfermedades por reglas. Madrid: Imprenta Real de la Gaceta, 1774.

Salvá y CAmpillo, Francisco. Análisis de la fiebre llamada vulgar e impropiamente amarilla, o vómito prieto. Barcelona: Viuda de A. Brusi e hijos, 1821.

Santamaría, Francisco. Diccionario de Mejicanismos. México D. F.: Porrúa, 1992.

SCHENEIDER, Konrad Victor. Liber de morbis capitis. Wittenberg: M. Henckell, 1669.

SOLARES, Francisco. El emperador político y politica de emperadores, II. Barcelona: Joseph Llopis, 1700.

TERreros, Esteban de. Diccionario castellano con las voces de las ciencias y las artes. Madrid: ArcoLibros, 1987 [1786-8].

TEsauro, Emmanuele. Filosofía moral. Lisboa: Antonio Craesbeeck, 1682.

VIDOS y Miró, Juan de. Medicina y cirugía racional y espagirica, II. Zaragoza: Pascual Bueno, 1720.

Virrey y Mange, Pascual F. Palma febril médico-práctica, II. Madrid: Antonio Sanz, 1756. 